\title{
Rugby and cervical spine injuries - has anything changed? A 5-year review in the Western Cape
}

\author{
Robert Neil Dunn, Dirk van der Spuy
}

Objectives. To review the incidence of all rugby-associated cervical spine injuries in the Western Cape and identify risk factors.

Methods. We reviewed case notes and X-rays of 27 male patients with rugby-related cervical spine injuries treated at the acute spinal injury (ASCI) unit at Groote Schuur Hospital from April 2003 to June 2008, and followed up with telephone interviews. Patient profile, rugby profile, subsequent injury management from the field to definitive surgery and neurological status on admission, discharge and followup using the American Spinal Injury Association (ASIA) classification were assessed.

Results. Average patient age was 25.3 years; $19 \%$ of them were scholars. The highest level of education among the adults was primary school in $70 \%$ of cases. Forwards and backs had the same injury rate. Most injuries occurred outside the metropole; more occurred in the tackling phase; $39 \%$ occurred during foul play; a third of players were not stabilised with a collar on the field; and $65 \%$ were taken to an inappropriate primary contact centre. A median of 10 hours elapsed before admission to the ASCI unit. Facet dislocations occurred in $59 \% ; 8$ presented neurologically complete and remained so; and 3 presented with residual sensation, with 2 improving to normal. Three presented as ASIA C improving to D, and all Ds improved to Es. Despite their injuries, 60\% said they would advise their sons to play rugby. Only $22 \%$ regretted playing.

Conclusions. Despite a reduction in cervical spine injuries in rugby in the Western Cape, the latter mostly occur outside the metropole, where levels of education are lower, foul play is more often associated with the injury, and rapid access to medical care is generally unavailable.

S Afr Med J 2010; 100: 235-238.
Rugby is increasingly popular in South Africa, with World Cup success following resumption of the country's international participation. New role models are continuously presented in the media, as a result of the numerous national and international tournaments. However, the risk of spinal column injury in rugby is perceived to be higher than in other sports, which can have a strongly deterrent effect on young and eager participants.

A 26-year review of spinal cord rugby injuries in the Western Cape ${ }^{1}$ found an alarmingly increasing incidence of injuries up to 1989 , escalating to 12 per annum. Many changes have since been made to the rules and in educating the players and coaches, culminating in SA Rugby's Boksmart programme. It is unclear whether a positive effect in terms of injury reduction has resulted.

Fuller $^{2}$ reports the risk of catastrophic injury at 0.8/100 000 in England and around 4/100 000 in Australia and New Zealand, and concludes that rugby risks are perceived to be higher than they really are, compared with other sports and activities such as motorcycling. We have attempted to address the paucity of South African data by retrospectively reviewing all spinal rugby injuries over the past 5 years ranging from quadriplegia

Division of Orthopaedic Surgery, University of Cape Town Robert Neil Dunn, MB ChB, MMed (Orth), FCS (SA) Orth

Department of Orthopaedic Surgery, Stellenbosch University, Tygerberg, W Cape Dirk van der Spuy, MB ChB

Corresponding author: R Dunn (robdunn@mweb.co.za) to those neurologically intact with unstable fractures at risk of secondary injury, presenting to a regional spinal injury unit.

\section{Methods}

The Western Cape regional spinal service at Conradie Hospital was relocated in 2003. The acute service was transferred to Groote Schuur Hospital (GSH), and the Acute Spinal Cord Injury Unit (ASCI) established. The responsibilities and referral patterns remained as at Conradie Hospital, with the ASCI accepting spinal column injuries from the whole Western Cape and occasionally from other provinces. Although the unit's priority remains patients with neurological deficit as a result of injury, those with unstable spine fractures and neurological preservation are also admitted as they require the same surgical and ward skills to prevent secondary cord injury. The patient profile should therefore be unchanged from that at Conradie Hospital.

A prospective database, maintained on all admissions to the ASCI unit, was examined for all spinal rugby injuries from April 2003 to June 2008. The database data, case notes and X-rays of 27 patients were reviewed, and the demographics, epidemiological results, medical management and outcomes were collated. The neurological deficits were quantified using the American Spinal Cord Injury Association (ASIA) scoring system which evaluates motor, light touch and pinprick deficits in spinal cord injuries.

Telephone interviews with 25 of the 27 injured established the following:

Rugby profile: Position, years playing rugby prior to injury, level of game, previous rugby injuries, subjective fitness level, awareness of spinal rugby injuries prior to accident. 
Circumstances of injury: Was game structured or not, referee qualified or not, mechanism of injury, was injury the result of illegal action and did the referee take action.

Injury management: Who managed the injury initially on the field, was neck stabilised on the field, did delay of more than 30 minutes before evacuation to hospital occur, what level of hospital first managed the injury, and time taken to reach the ASCI unit.

Outcome: Improvement after discharge, whether returned to playing rugby and returned to work, whether regretting playing rugby, and whether they will advise their children not to play rugby.

Ethical approval was obtained from the Divisional Research Committee and Ethical Committee of the Health Sciences Faculty of the University of Cape Town.

\section{Results}

All patients were male. The average age at the time the injury was $25.3(16-46 \pm 8)$ years. Of the 20 adults, the highest level of education was high school in $4(25 \%)$, primary school in 14 (70\%), and unknown in 2.

There was an even distribution of injuries between forwards and backline players; 19\% of injuries occurred in school games and the rest at club rugby; no patients had previous spinal injuries; $20 \%$ had a previous significant non-spinal injury varying from shoulder injuries to fractured limbs; and $71 \%$ considered themselves to have been fit at the time of injury (Table I).

\section{Geographical distribution of injuries}

Most injuries occurred outside the Cape Town metropolitan area. Of the 3 injuries occurring in the metropole, 2 occurred in Langa, which may be significant as games in this area may not be well controlled (Tables II and III).

Of the above injuries, $8(30 \%)$ were the result of illegal behaviour, and the referee took some form of action in $75 \%$ of cases, including a penalty or sending the offending player to the 'sin bin'.

\section{Injury management on field}

These data were derived from the patients' accounts of the incidents. In 9 players, the cervical spine was not stabilised in any way immediately after the on-field injury. A delay of about 30 minutes or more occurred before evacuation from the field occurred in 20 (77\%) of the cases. A primary health care facility

\begin{tabular}{lcc}
\multicolumn{3}{l}{ Table I. Incidence of injury per player position } \\
\hline Position & No. of injuries & $\%$ \\
\hline Scrumhalf & 6 & 22 \\
Flank & 3 & 11 \\
Prop & 6 & 22 \\
Centre & 3 & 11 \\
Wing & 3 & 11 \\
Hooker & 3 & 11 \\
Lock & 1 & 4 \\
Unknown & 2 & 7 \\
\hline
\end{tabular}

\begin{tabular}{lcc} 
Table II. Distribution of injuries per region & \\
\hline District/metropole & No. of injuries & $\%$ \\
\hline Cape Town metropole & 3 & 11 \\
South-western Cape & 10 & 37 \\
Boland & 7 & 26 \\
Swartland & 3 & 11 \\
West Coast & 2 & 3 \\
Eastern Cape & 2 & 3 \\
\hline
\end{tabular}

Table III. Phases of play where injury occurred

\begin{tabular}{lcc}
\hline Phase of play & No. of injuries & $\%(N=27)$ \\
\hline Scrum & 10 & 37 \\
Maul or ruck & 2 & 7 \\
Tackling & 14 & 52 \\
Unknown & 1 & 4 \\
\hline
\end{tabular}

was the first contact point in $65 \%$ of the cases, most of which did not have $\mathrm{X}$-ray facilities.

\section{Management of the patient at the ASCI unit}

There was an average delay of 42.4 (3 - 384 \pm 79$)$ hours from injury to admission at the ASCI unit. The average was skewed by 3 cases of delayed presentation greater than 5 days. The median was 10 hours. The worst delays occurred outside the Western Cape but were subsequently referred to our unit for definitive management. The patient who was longest delayed was admitted to a secondary hospital where the dislocation was not reduced during the 16-day admission and only definitively treated on arrival at the ASCI unit. Another 168hour (7 days) delay occurred with a patient who injured his neck in Oudtshoorn. There was no urgency to transfer as he was neurologically intact and placed in traction until transport and a bed in the ASCI unit was available. These data were skewed by some patients having waited for a bed in the ASCI unit at other secondary or tertiary units, and therefore not necessarily reflecting poor transfer infrastructure. Patients all 'compete' for a bed on the basis of medical priority rather than first-come-first-served.

The distribution of injuries was: unifacet dislocation -10 $(37 \%)$, bifacet dislocation $-6(22 \%)$, flexion compression -3 (11\%), dens type II fracture $-3(11 \%)$, and spinal cord injury without radiological abnormality (SCIWORA) $-5(19 \%)$. Of the 16 distractive flexion injuries (facet dislocations), 3 underwent immediate successful closed reduction; 5 failed closed reduction; reduction was not attempted in 3 owing to the delay between injury and presentation; and 5 proceeded immediately to open reduction surgery. Six patients were managed nonoperatively with traction and external orthosis. The other 21 underwent surgery comprising 16 anterior cervical decompression and instrumented fusion procedures and 4 posterior fusions; 3 of the 16 anterior procedures included corpectomy. Posterior fusions were generally utilised in delayed presentation where open facet reduction was required.

The ASIA admission neurological status is depicted on the vertical axis of the table and the outcomes distributed over the horizontal axis (Table IV). Complete motor and sensory loss is 


$\begin{aligned} & \text { Table IV. Admission versus outcome by ASIA neurological } \\
& \text { status }\end{aligned}$
\begin{tabular}{llllll} 
& \multicolumn{5}{c}{ Outcome status } \\
\cline { 2 - 5 } & A & B & C & D & E \\
\hline $\begin{array}{l}\text { Admission } \\
\text { status } \\
\text { A }\end{array}$ & & & & & \\
B & 8 & & & & \\
C & & 1 & 3 & 2 \\
D & & & & \\
E & & & & 6 \\
\hline
\end{tabular}

A, residual sensation is B, power less than $3 / 5$ in at least $50 \%$ of distal muscle groups is $C$, power greater than $3 / 5$ in at least $50 \%$ of distal muscle groups is $\mathrm{D}$, and $\mathrm{E}$ is normal. Accordingly, 8 patients presented as complete; none of these improved, and this group included the 2 who died. Strikingly, two of the Bs (i.e. sensory preservation) improved to normal. All the C and D patients improved by one grade.

Accordingly, 14 catastrophic injuries (ASIA A - C) occurred; of these patients 2 died, and 2 improved to normal and 3 to near-normal status. At follow-up there were 7 surviving players with residual catastrophic neurological deficit. The more severe neurological injuries did not correlate with injury type or age; they had relatively rapid access to definitive care with an average delay of $7.8(3-12 \pm 2.7)$ hours.

\section{Players' remarks about their injuries}

Only 5 (19\%) players were concerned about spinal cord injuries before their injury; 16 (60\%) of injured players recommended their sons to play rugby and, surprisingly, $71 \%$ of those surviving with catastrophic injuries said the same. Only 6 $(22 \%)$ patients regretted that they had played rugby.

\section{Discussion}

Our study indicates an annual cervical spine rugby injury incidence in the Western Cape of 5.4, with 1.8 regarded as catastrophic in the sense of permanent quadriplegia/ quadraparesis. The former figure is well down from 8 per annum in the 1980s, and 12 in $1989 .{ }^{1}$ It is difficult to determine whether additional injuries occur but are managed outside the state-funded system. At the time of the Kew study, ${ }^{1}$ there were few private hospitals, and almost all injuries were most probably managed at Conradie Hospital. Now there are many private options for acute care. However, few surgeons seem willing to take on the responsibility of acute spinal injuries, especially in young sportsmen, as evidenced by referrals to the senior author's practice. This holds especially true for the neurologically impaired sportsman requiring urgent intervention. The only private rehabilitation alternative is the UCT private hospital, also located at Groote Schuur Hospital (GSH). As the GSH spine consultants also cover this facility, they are aware of the injuries admitted. Only one acute cervical rugby injury was managed there during this period, and it is included in this cohort. Therefore, it appears that this paper reflects the bulk of the serious injuries but may under-report the incidence of lesser injuries, especially where there are no neurological deficits or surgery required.
Overall, it appears that the incidence of cervical rugby injuries in the Western Cape has fallen since the 1980s. The peak in 1989 returned to an average of 8 per annum, as reported for the years $1990-1997,{ }^{3}$ which showed a reduction in the proportion of schoolboys injured (which had been 20\%) compared with the earlier figure of $31 \%$. These figures are consistent with our findings.

An overall 1.4 spinal cord injuries per annum per 100000 New Zealand rugby players has been reported. ${ }^{4}$ This figure reduced to 0.7 since 2001 and is claimed to coincide with the introduction of a local education programme. Care is necessary with this interpretation as there have been simultaneous law changes reducing the number of scrums per game by $40 \% 5,6$ - these being the major injury risk. Following 12 English Premiership clubs for two seasons, an incidence of 10.9 spinal column injuries per 1000 player-match-hours was found. ${ }^{7}$ None was catastrophic, but 3 were career-ending. Unfortunately, there are no reliable figures for rugby players in South Africa to use for comparison.

Despite changes to the rules, there seems little change in the phases of play where injuries occur. Kew's earlier SA data shows scrums/rucks/mauls at $40 \%$, tackles at $57 \%$ and foul play at 3\%. Noakes's report on the SA data of the 1990s indicates tackles at $52 \%, 25 \%$ in rucks/mauls and $23 \%$ in scrums. Our data indicate far higher scrum incidence at 37\%, rucks $/$ mauls at $7 \%$ and tackles at $52 \%$. Comments have been made about reduced scrum injury rates ${ }^{4}$ and confirmation of the tackle as the culprit, albeit in small numbers. ${ }^{7}$ South Africa is applying similar education and law changes yet the distribution has not changed. However, intervention should be seen to be effective as long as the absolute number of injured is reduced.

It appears that the SA injury rate is still unacceptably high, even if the catastrophic incidence is lower. The most noticeable variable is geographic distribution. The vast majority of our injuries occur outside metropoles and where the level of patients' formal education is also low, suggesting that the less regulated games carry the higher risk. In the metropole, there may be better coaching and referee control of play, and the players may be more disciplined, possibly by virtue of higher education. This may be borne out by how few cervical injuries are sustained at professional level, where one expects the game to be far tougher. Consistent with the possibility of inadequate game control is the high level of associated foul play $-32 \%$ of the injuries resulted from foul play. The referee only took action in $75 \%$ of cases, in the form of a penalty or carding the offender.

As injuries tend to occur outside major centres, delay to definitive care is understandable. We report a median time of injury to definitive care of 10 hours. If neurologically intact, there is no urgency and patients may be managed initially at secondary units in traction or with spinal precautions. However, if a neurological deficit is present, it becomes urgent to reach a centre where appropriate investigations, especially MRI, and surgical facilities/skills are available; this is generally not happening. Of those sustaining serious neurological injury (ASIA A, B or C), only 1 patient reached 
definitive care within 4 hours, and another 8 players in 8 hours or less. Newton (personal communication) has constantly motivated for urgent closed reduction of distractive flexion injuries within 4 hours, so maintaining an increased chance of neurological recovery. Although unpublished, Newton subanalysed the Kew data from the Conradie unit and maintains that the Frankel As reduced within 4 hours had a much higher incidence of neurological recovery. Bifacet dislocation is the one cervical injury that shows evidence of benefit from earlier decompression; unfortunately, this is not being achieved.

On-field management remains sub-optimal, with a third of patients not being primarily stabilised with a collar on the field. There was also a $>30$-minute delay before removal from the rugby site in $77 \%$ of cases. Again, this fits in with peripheral, less wealthy clubs, possibly without attendant paramedic staff. The first medical contact was inappropriate in $65 \%$ of cases, as even basic investigations such as X-rays were unavailable.

Once in the ASCI unit, definitive management and surgery were performed on the basis of the medical urgency. There is no urgency to reduce or stabilise surgically if the patient is already a few days post-injury and waiting on the nextavailable list. If the patient presents within a few hours, an attempt is made to expedite management. Consequently, 5 patients went to theatre immediately, undergoing open reduction and instrumented fusion, obviating the risks of disc prolapse into the spinal canal with closed reduction, and avoiding the delay in waiting for an MRI. We prefer MRI prereduction or surgery as it is usually available without too much delay. However, should MRI represent a significant delay, the patient may be better off reduced with the possibility of better neurological outcome than the small risk of the disc being swept back into the canal.

In terms of neurological improvement, there was excellent return to normal function in 2 ASIA Bs, i.e. presented with only some sensory preservation, and no motor function. All the Cs and Ds improved by one grade. Unfortunately, none of the As (complete motor and sensory loss) improved, and 2 died. This is contrary to Newton's unpublished data that have been used to motivate for rapid access to care, i.e. the 4-hour window of opportunity. All neurological assessments are subjective and related to the patient's co-operation and the examining doctor's diligence. In the initial acute phase, the patient is bewildered and distressed and residual peri-anal sensation may not be volunteered. The same is true for the doctor who is dealing with an emotionally charged clinical situation. The first medical contact is often a junior or a generalist whose priority is to transfer the injured player to a hospital that can deal with the injury. This situation lends itself to a superficial examination that may miss residual sensation and cause the patient to be classed as an A rather than a B, with vastly differing prognosis. Spinal shock may also disguise the fact that there is residual sensation, which only becomes evident on re-examination. Of 358 cases presenting as neurologically complete initially, 30 subsequently improved. ${ }^{8}$ This potential recovery from complete injury is thought to be even more likely in low-velocity rugby injuries, where the cord is compressed rather than disrupted anatomically.

It is interesting how few patients considered spinal cord injury a risk, and that $60 \%$ of them will nevertheless recommend their sons to play rugby. Another consideration is that skilful young players often play in higher age groups - a situation which is more likely to occur in smaller peripheral clubs.

As the number of rugby players is not known, the reported number of spinal injuries does not necessarily reflect an increased incidence in the peripheral clubs. However, the findings in our study provide a focus for involved parties towards improving the safety of the game and maximising recovery in the few unfortunate, injured players.

1. Kew T, Noakes TD, Kettles AN, Goedeke RE, Newton DA, Scher AT. A retrospective study of spinal cord injuries in Cape Province rugby players 1963-1989. S Afr Med J 1991; 80: 127-133. . Fuller CW. Catastrophic injury in rugby union: is the level of risk acceptable? Sports Med 2008; 38(12): $975-986$

3. Noakes TD, Jakoet I, Baalbergen E. An apparent reduction in the incidence and severity of spinal cord injuries in schoolboy rugby players in the Western Cape since 1990. S Afr Med J 1999; 89: 540-545.

4. Quarrie KL, Gianotti SM, Hopkins WG, Hume PA. Effect of nationwide injury prevention programme on serious spinal injuries in New Zealand rugby union: ecological study. $B M$, 2007: 334; 1150-1153.

5. Silver JR. The impact of the 21st century on rugby injuries. Spinal Cord 2002; 40: 552-559.

6. Quarrie KL, Cantu RC, Chalmers DJ. Rugby union injuries to the cervical spine and spinal Quarrie KL, Cantu RC, Chalmers DJ.
cord. Sports Med 2002; 32(10): 633-653.

7. Fuller CW, Brooks JH, Kemp SP. Spinal injuries in professional rugby union: a prospective cohort study. Clin J Sport Med 2007; 17(1): 10-16.

8. Kenneth A, Gerhart MS. Spinal cord injury outcomes in a population-based sample. J Trauma 1991; 31(11): 1529-1535

Accepted 27 August 2009. 